\title{
ON A PROBLEM OF PAPAKYRIAKOPOULOS
}

\author{
BY ELVIRA STRASSER RAPAPORT
}

Communicated by Lipman Bers, January 29, 1963

Let $P^{*}$ be the group generated by the symbols $a, b, c_{2}, d_{2}, c_{3}, d_{3}$, $\cdots, c_{0}, d_{0}$ and subject to the relation

$$
G^{*}: a b a^{-1} b^{-1} c_{2} d_{2} c_{2}^{-1} d_{2}^{-1} \cdots c_{\sigma}^{-1} d_{\sigma}^{-1}=1 .
$$

Let $w^{*}$ be an element of $P^{*}$ and $N^{*}$ the normal subgroup the word $R^{*}=w^{*} a w^{*-1} a^{-1}$ generates in $P^{*}$.

The question whether the factor group $P^{*} / N^{*}$ is torsionfree (has no element of finite order) arose in connection with the Poincare conjecture [4]. I shall sketch a proof of the fact that $P^{*} / N^{*}$ is torsionfree (a detailed proof will appear elsewhere).

An extension $E$ of a torsionfree group $H$ by the free cyclic group is torsionfree, so I will present $P^{*} / N^{*}$ as such an extension. Consider the normal subgroup $H^{*}$ which the symbols $c_{2}, d_{2}, \cdots, c_{g}, d_{0}$ and $b$ generate in $P^{*} / N^{*}$; its factorgroup is the free cyclic group generated by $a$, so $P^{*} / N^{*}$ is an extension of $H^{*}$ by the free cyclic group generated by $a$. I shall show now that $H^{*}$ is torsionfree.

The presentation below of $H^{*}$, from which the required property is clearly seen, is based on the infinite set of generating symbols $b_{k}$, $c_{i k}, d_{i k}$, where, for every integer $k$,

$$
b_{k}=a^{-k} b a^{k}, \quad c_{i k}=a^{-k} c_{i} a^{k}, \quad d_{i k}=a^{-k} d_{i} a^{k} .
$$

The left-hand side of the defining relation $G^{*}$ given above for $P^{*}$, written in terms of these symbols, becomes

$$
G_{0}=b_{-1} b_{0}^{-1} c_{20} d_{20} \cdots \cdot c_{00}^{-1} d_{g 0}^{-1}
$$

and the conjugates $a^{-k} G^{*} a^{k}$ become

$$
G_{k}=b_{k-1} b_{k}^{-1} c_{2 k} d_{2 k} \cdots c_{g k}^{-1} d_{g k}^{-1} .
$$

The left hand side of the defining relation $R^{*}=1$ given above for $P^{*} / N^{*}$ can also be written by means of these symbols: there is an integer $h$ for which $w^{*} a^{h}$ can be so written (namely when $w^{*} a^{h}$ contains the symbol $a$ to exponent sum zero), say, in the form

$$
v_{0}=v\left(b_{s}, \cdots, c_{2 t}, \cdots, d_{g u}, \cdots\right),
$$


so that, if we define

$$
v_{k}=v\left(b_{s+k}, \cdots, c_{2, t+k}, \cdots, d_{g, u+k}\right),
$$

for integral $k$,

$$
v_{-1}^{-1}=v^{-1}\left(b_{s-1}, \cdots, c_{2, t-1}, \cdots, d_{g, u-1}\right)
$$

is the form taken by $a\left(a^{-h_{2} w^{*-1}}\right) a^{-1}$, whence

becomes

$$
R^{*}=w^{*} a w^{*-1} a^{-1}=w^{*} a^{h} a\left(a^{-h} w^{*-1}\right) a^{-1}
$$

$$
R_{0}=v_{0} v_{-1}^{-1}
$$

and $a^{-k} R^{*} a^{k}$ becomes

$$
R_{k}=v_{k} v_{k-1}^{-1} \text {. }
$$

Letting $x_{i j}$ stand for the symbols $c_{i j}$ and $d_{i j}$, we get the following presentation $H$ of the subgroup $H^{*}$ of $P^{*}$ :

$$
H=\left(x_{i j}, b_{j} ; G_{j}, R_{j}, i: 2, \cdots, g, j: 0, \pm 1, \cdots\right) .
$$

If the two sets of words $\left(G_{j}, R_{j}, j: 0, \pm 1, \cdots\right)$ and $\left(G_{j}, A_{j}, j\right.$ : $0, \pm 1, \cdots)$ generate the same normal subgroup in the free group on the symbols of $H$, then

$$
H=\left(x_{i j}, b_{j} ; G_{j}, A_{j}, i: 2, \cdots, g, j: 0, \pm 1, \cdots\right) .
$$

I will pick the set $A$ to suit my purpose.

If $P^{*} / N^{*}$ has torsion, so does the group $H$ [1]. I shall express $H$ as the free product of isomorphic groups $H_{r}, r: 0, \pm 1, \cdots$, with a free subgroup amalgamated between $H_{r}$ and $H_{r+1}$. If $H$ has torsion, so does $H_{r}[1 ; 2]$. The latter will however prove torsionfree.

Using combinatorial arguments, it can be shown that there is an $A_{0}$ (cyclically reduced, i.e. not of the form $z B z^{-1}$ for $z \neq 1$ ) with the following properties:

1. If $A_{0}$ contains any $b_{j}$-symbol, then it contains only $b_{0}$;

2. $A_{0}$ actually contains some $x_{i j}$-symbols, and either only $j=0$ occurs for these, or else $A_{0}$ contains an $x_{i j}$ with $j$ at most zero and also an $x_{i^{\prime} j^{\prime}}$ with $j^{\prime}$ at least one;

3. $A_{0}$ is not a formal power $B^{k}$ unless $k= \pm 1$.

Suppose that among the subscripts $j$ of the $x_{i j}, i: 2, \cdots, g$ in $A_{0}$ the least is $u$, the largest $v$. Then, by property 2 , above, either $u=v=0$, or $u \leqq 0, v \geqq 1$.

Let $r$ be some integer. Define the groups $H_{r}$ and $S_{r}$ as follows:

$$
H_{r}=\left(x_{i, j+r}, b_{r} ; A_{r}, i: 2, \cdots, g, j: u, \cdots, v\right)
$$


and $S_{r}$ the subgroup of $H_{r}$ generated by a set of elements $x_{r}$ in $H_{r}$ such that when $u \leqq 0<v$ for $A_{0}$

$$
x_{r}=\left(x_{2, u+r+1}, x_{3, u+r+1}, \cdots, x_{g, u+r+1}, \cdots, x_{g, v+r}, b_{r}^{-1}\right)
$$

and when $u=v=0, x_{r}$ is the last element $b_{r}^{-1}$ above.

Similarly, define the groups

$$
H_{r+1}=\left(x_{i, j+r+1}, b_{r+1} ; A_{r+1}, i: 2, \cdots, g, j: u, \cdots, v\right)
$$

and $T_{r}$ the subgroup of $H_{r+1}$ generated by a set of elements $X_{r}$ in $H_{r+1}$ such that when $u \leqq 0<v$ for $A_{0}$

$$
\begin{aligned}
X_{r}=\left(x_{2, u+r+1}, x_{3, u+r+1}, \cdots, x_{g, u+r+1}, \cdots, x_{g, v+r},\right. \\
\left.b_{r+1}^{-1} c_{2, r+1} d_{2, r+1} c_{2, r+1}^{-1} d_{2, r+1}^{-1} c_{3, r+1} \cdots d_{g, r+1}^{-1}\right)
\end{aligned}
$$

and, when $u=v=0, X_{r}$ is the last element listed above.

According to the Freiheitssatz [3], in a group on one cyclically reduced defining relation, every subset of the generating symbols gives rise to a free subgroup provided not every symbol present in that defining relation occurs in the set in question. This condition holds for the symbols of $x_{r}$ in $H_{r}$ and the symbols $X_{r}$ in $H_{r+1}$. Therefore, properties 1 and 2 of $A_{0}$, inherited by $A_{r}$ and $A_{r+1}$, imply that the subgroups $S_{r}$ and $T_{r}$ are free groups isomorphic under the mapping that associates the two sets of elements $x_{r}$ and $X_{r}$ in the order given above.

Because of property 3 of $A_{0}$, inherited by $A_{r}$, the group $H_{r}$ is torsionfree [1], and so is the free product of $H_{r}$ and $H_{r+1}$ with amalgamation of $S_{r}$ and $T_{r}$ and, finally, the free product of all $H_{r}$ with the (infinitely many) amalgamations of $S_{r}$ and $T_{r}, r=0, \pm 1$, $\pm 2, \cdots$.

Inspection of the defining relations $G=1$ shows that the last named group is $H$. Thus $P^{*} / N^{*}$ is torsionfree.

\section{BIBLIOGRAPHY}

1. A. Karrass, W. Magnus, and D. Solitar, Elements of finite order in groups with a single defining relation, Comm. Pure Appl. Math. 13 (1960), 57-66.

2. A. G. Kurosh, The theory of groups. I, Chelsea, New York, 1956, p. 32.

3. W. Magnus, Ueber diskontinuierliche Gruppen mit einer definierenden Relation (der Freiheitssatz), J. Reine Angew. Math. 163 (1930), 141-165.

4. C. D. Papakyriakopoulos, A reduction of the Poincare conjecture to other conjectures. II, Bull. Amer. Math. Soc. 69 (1963), 399-401.

Polytechnic Institute of Brooklyn 